\title{
A retrospective analysis of the InterTan nail and proximal femoral nail anti-rotation in the treatment of intertrochanteric fractures in elderly patients with osteoporosis: a minimum follow-up of 3 years
}

\author{
Hui Zhang ${ }^{1 *+}$, Xiaoxiao Zhu ${ }^{2+}$, Genwang Pei ${ }^{3+}$, Xianshang Zeng ${ }^{4+}$, Nan Zhang ${ }^{5+}$, Ping X ${ }^{6+}$, Deng $\mathrm{Chen}^{7 \dagger}$,
} Weiguang $\mathrm{Yu}^{4+}$ and Xinchao Zhang $8^{8^{*}}$

\begin{abstract}
Background: The study aims to compare the long-term functional and radiographic outcomes of two devices for the treatment of primary intertrochanteric fractures (IFs, OTA 3.1A2.1-A2.3) in elderly patients with osteoporosis.

Methods: Between December 2010 and August 2014, 332 elderly osteoporosis patients with IFs (OTA 3.1A2.1-A2.3) fixed by an InterTAN nail (IT) or a proximal femoral nail anti-rotation (PFNA) device were retrospectively evaluated. Follow-up occurred 1, 3, 6, and 12 months postoperatively and every year thereafter. Mortality was recorded. Patient-related functional and radiographic outcomes were compared. The primary endpoint was the long-term radiographic outcomes. The secondary endpoint was the long-term functional outcomes.

Results: A total of 283 patients (283 hips) with osteoporosis (IT, $n=144$; PFNA, $n=139$ ) were evaluated with a mean follow-up period of 38.8 months (range, 36-43 months). No between-group significant differences were noted in the patient demographics, operation variables, and postoperative Harris Hip Score. More radiographic complications were noted in terms of screw cut-out, femoral shaft fracture distal or around the tip of the main nail, and varus collapse of the femoral head in the PFNA group compared with that in the IT group $(P<0.05)$.
\end{abstract}

Conclusion: For osteoporotic IFs (OTA 3.1A2.1-A2.3) in elderly patients, the use of IT aids in decreasing radiographic complications, but the between-group functional outcomes showed no significant difference.

Keywords: Intertrochanteric fracture, InterTAN nail, Proximal femoral nail anti-rotation, Harris Hip Score

\section{Background}

With the aging population, the incidence of intertrochanteric fractures (IFs) is increasing [1-3]. Because elderly patients have comorbidities, the mortality rate from IFs in these patients is 12 to $41 \%$ within 6 months $[4,5]$. Studies have shown that early surgical intervention

\footnotetext{
*Correspondence: 13751886190@126.com; zhangxc666@aliyun.com ${ }^{\dagger}$ Equal contributors

'Emergency Department, The First Affiliated Hospital of Sun Yat-sen University, Huangpu East Road No. 183, Huangpu District, Guangzhou, Guangdong 510700, China

${ }^{8}$ Department of Orthopaedics, Jinshan Hospital, Fudan University, Longhang Road No. 1508, Jinshan District, Shanghai City 201508, China

Full list of author information is available at the end of the article
}

(within $24 \mathrm{~h}$ ) can significantly reduce mortality [5]. Intramedullary fixation, with the advantages of withstanding the stress of the axis shift, good antifatigue performance, smaller incisions, and less damage to the local blood supply, is the most current treatment for IFs $[2,6]$.

Most elderly patients following IFs, without osteoporosis, are treated surgically (IT or PFNA) and have good surgical results $[7,8]$. Nevertheless, some elderly patients with osteoporotic IFs undergo IT or PFNA processes and are most likely to have poor surgical results $[9,10]$. Data from recent studies have confirmed that an increasing number of elderly patients have been presenting 
with osteoporotic IFs [11-16]. In China, for example, the number of fractures attributable to osteoporosis will increase between 2017 and 2040 by approximate 129\% if the fracture rate $(5.6 \%)$ remains constant and is estimated to be 45.0 million. The prevalence of osteoporotic fractures in Europe in 2017 was estimated at 25.0 million. It may, therefore, be very meaningful to know what happens to this group of patients.

The aim of this study was to compare the clinical and radiographic outcomes of InterTAN nail (IT) and proximal femoral nail anti-rotation (PFNA) in the management of IFs (OTA 3.1A2.1-A2.3) in elderly patients with osteoporosis with a 3-year minimum follow-up.

\section{Methods}

\section{Study population}

This study is a retrospective review from a trauma database (Jinshan Hospital, Fudan University). The variability tested of descriptive characteristics of patients in Table 2 was statistically non-significant between the groups $(P>0.05)$. Patient age was divided into five categories: 65-69, 70-79, 80-89, 90 years, or older. Chronic illness burdens before IFs were similar for the two groups as per comparison of the number of comorbidities. Osteoporosis was defined as a bone mineral density (BMD) T-score value $\leq-2.5$ at the contralateral femoral neck.

\section{Inclusion and exclusion criteria}

The inclusion criteria were as follows: freshly closed IFs (OTA 3.1A2.1-A2.3) following a fall, age ranging from 65 to 92 years, a BMD T-score value $\leq-2.5$ at the contralateral femoral neck, and IT or PFNA fixation (IT, diameter: lag screw, $11 \mathrm{~mm}$; compression screw, $7 \mathrm{~mm}$; composite screw, $15.5 \mathrm{~mm}$; length, normal; number of proximal/distal screws, 2/1, Smith \& Nephew, Memphis, Tennessee; PFNA, proximal diameter, $16.5 \mathrm{~mm}$; distal diameter, $9-10 \mathrm{~mm}$; length, 240 or $300 \mathrm{~mm}$; number of proximal/distal screws, $1 / 1$; valgus curvature, $5^{\circ}$, Synthes, Solothurn, Switzerland). Exclusion criteria included multiple fractures, fracture secondary to cancer or major trauma, hip fracture during the previous calendar year, sub-trochanteric fracture or femoral neck fracture, and an American Society of Anesthesiologists (ASA) score of IV or V.

\section{Surgical methods}

All of the patients with osteoporosis received an intravenous injection of cefazolin sodium pentahydrate $(2.0 \mathrm{~g}) 30 \mathrm{~min}$ before surgery. The patients were placed on a traction operation bed and received general anesthesia. The limb was neutral. Closed traction reduction was performed under an X-ray machine for all of the patients. The point of needle insertion was slightly medial to the exact tip of the greater trochanter. The tip-apex distance (TAD) was limited to approximately $20 \mathrm{~mm}$. No bone grafting was carried out in any of the cases. In the PFNA group, a small incision was made, and the main nail was rotated into the medullary cavity to stabilize the fracture followed by the locator, locking the nail proximally or distally. In the IT group, the installation of compressing intramedullary interlocking nails was performed. The pressurization effect was confirmed. Next, the distal locking screws were installed. All of the surgeries were carried out at our institution by orthopedic surgeons. The technique was identical to that described by Mereddy et al. [17] for PFNA and Ruecker et al. [18] for IT.

\section{Postoperative management}

Passive movement of the quadriceps femoris and ankle joint was performed immediately after surgery. A pneumatic drive pump promoting backflow of the lower limbs was utilized. A once-daily subcutaneous injection of Clexane (4000 AXa IU, Shanghai, China) for the prevention of lower limb venous thrombosis was carried out as early as $6 \mathrm{~h}$ postoperatively for seven subsequent days. Twice-daily cefuroxime sodium $1.5 \mathrm{~g}$ was used beginning the day before surgery and for three subsequent days. On the third day after surgery, continuous passive motion (CPM; Smith \& Nephew, Shanghai, China) was applied to promote the rehabilitation of hip joint function. Postoperative X-rays were used to examine fracture stability. The rehabilitation protocol was identical, and the patients were mobilized on the first postoperative day. Partial weight-bearing as tolerated or restricted weight-bearing was allowed according to the surgeon's recommendation on the following day.

\section{Method of assessment}

Follow-up occurred 1, 3, 6, and 12 months postoperatively and every year thereafter. The primary endpoint measured was the radiographic outcomes which were obtained at each visit. Major changes of the implant were noted. Electronic medical records and digital radiographs were reviewed by two of us (WY and HZ) to collect all variable outcomes which were assessed by a review (WY). The secondary endpoint was patientrelated functional outcomes which were evaluated based on the Harris Hip Score (HHS) by a surgeon-assessor (XCZ). Malunion was defined as less than $50 \%$ contact between the proximal and distal fragments or collodiaphyseal angle (CCD) of less than $120^{\circ}$. Bone union was defined by the following radiographic parameters: restoration of cortical continuity, loss of a clear fracture line, and presence of callus. Non-union which means nonunion of the fracture itself, not that of the third fragment, was defined as the state in which disturbed consolidation of a fracture that needs further surgical 
intervention or a prolonged healing time of more than 12 months or more. TAD was measured based on the description of Li et al. [19]. A literature review was then conducted to identify similar studies and to compare the functional and radiographic outcomes of two devices.

\section{Statistical analysis}

All statistical analysis was performed using SPSS (SPSS statistical package; version 22.0.0). Case characteristics were summarized using descriptive statistics, including the mean (SD), or median (minimum-maximum) for continuous variables and the frequency (percent) for categorical variables. Independent samples $t$-test and chi-square test were used to measure the differences in bi-variate analyses. For all comparisons, statistical significance was assigned at $P<0.05$.

\section{Results}

\section{General data comparison}

From December 2010 to August 2014, 283 patients (283 hips) with osteoporotic IFs (OTA 3.1A2.1-A2.3) stabilized with IT or PFNA devices were enrolled in this study (Figs. 1 and 2, Table 1). The average age was 76.1 years (Table 2). There was no perioperative mortality. During the next follow-up period, there were 41 deaths recorded. No between-group significant differences were noted in terms of the average amount of bleeding, average operation time, and average length of stay. Perioperative medical complications occurred in 20 cases: 7 cases of recurrence of heart disease, 3 cases of pulmonary infection, 2 cases of urinary retention, 4 cases of cerebral infarction, and 2 cases of gastrointestinal dysfunction. The symptoms improved after consultation with relevant departments. The mean follow-up period was 38.8 months (range, 36-43 months) (Tables 3).

\section{Clinical outcome}

HHS was used to evaluate the functional outcome. In the IT group, the mean HHS at the last follow-up was $72.2 \pm 7.27$. In the PFNA group, the mean HHS was $72.4 \pm 7.20$ at the last follow-up. With regard to the thigh pain in the distribution of the lateral cutaneous nerve of the thigh following surgery in the two cohorts, there was a lower complication rate in IT-treated patients compared with PFNA-treated patients (3.5 vs. $9.4 \%, P=0.043)$. Although there was notable difference in thigh pain between groups, no between-group significant difference in HHS was observed at each follow-up.

\section{Radiographic outcome}

Between-group significant differences were noted in fixation failure (cut-out, femoral shaft fracture distal or around the tip of the main nail, varus collapse of the femoral head), which is showed in Table 4.
The incidence of fixation failures in IT-treated patients was significantly lower than PFNA-treated patients (cutout 2.1 vs. $7.9 \%, P=0.024$; femoral shaft fracture distal or around the tip of the main nail, 2.1 vs. $8.6 \%$, $P=0.014$; varus collapse of the femoral head, 0.7 vs. $5.8 \%, P=0.020$, respectively). The average TAD was similar between the IT-treated and PFNA-treated patients $(19.9 \mathrm{~mm}$ [range, $17-25 \mathrm{~mm}$ ] vs. $20.2 \mathrm{~mm}$ [range, $16-23 \mathrm{~mm}], P=0.144)$. Among IT-treated patients, fixation failed in 7 fractures (4.8\%), which consisted of 3 cut-outs, 1 varus collapse of the femoral head requiring further surgery intervention as of the 2-year follow-up, 1 femoral shaft fracture distal to the tip of the main nail, and 2 femoral shaft fractures around the tip of the main nail requiring the revision surgeries 1.5 years after surgery. Three patients with failed internal fixation received surgical intervention (arthroplasty) secondary to varus collapse with a screw cut-out. Three patients developed varus collapse and failed to have further surgery. One patient with varus collapse without screw cut-out refused further surgical intervention due to the lack of economic ability. Among PFNA-treated patients, fixation failed in 31 fractures (22.3\%), which consisted of 11 cutouts, 8 varus collapses of the femoral head requiring further surgical intervention as of the 3-year follow-up, and 4 femoral shaft fractures distal to the tip of the main nail and 8 femoral shaft fractures around the tip of the main nail requiring revision surgeries 2 years after surgery. There was significant difference with respect to the mean time to failure between IT-treated and PFNAtreated cohorts (18 months [range, 8-19 months] vs. 8 months [range, $2-9$ months], $P=0.000$ ).

\section{Discussion}

The most important finding of the current research was that the use of IT aids in decreasing radiographic complications, but the between-group functional outcomes showed no significant difference.

Increasingly, PFNA devices are being used to manage IFs [19]. Compared with IT devices, most previous studies have shown a higher radiographic complication rate using PFNA devices $[3,20]$. However, in those studies, there was no significant difference in the rate of varus collapse between the PFNA and IT devices. In a series of 225 cases with IFs fixed with a PFNA or IT device, investigators described that varus collapse occurred in 4.9 or $5.5 \%$ of cases, respectively $(P=0.95)$ [14]. Inconsistent with previous reports, in this study, the PFNA was related to radiographic complications such as varus collapse (5.8\%), femoral shaft fracture distal or around the tip of the main nail (8.6\%), and screw cut-out (7.9\%). Our findings are comparable to the results published in other studies [15, 21-23]. Nevertheless, the management 


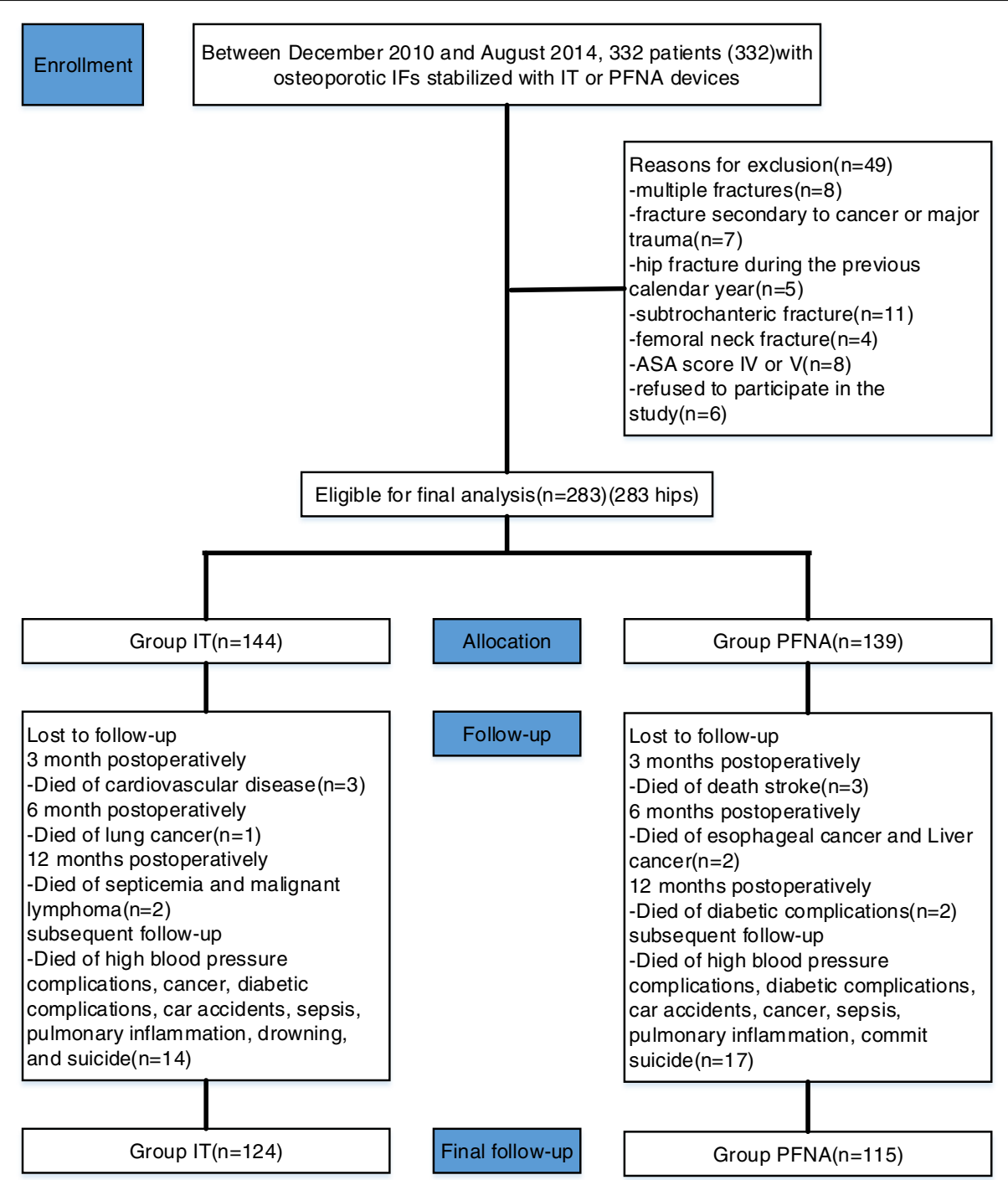

Fig. 1 Flow diagram demonstrating methods for identification of studies to assess elderly osteoporosis patients with IFs (OTA 3.1A2.1-A2.3) fixed by an InterTAN nail (IT) or a proximal femoral nail anti-rotation (PFNA) device

of IFs remains controversial, particularly in utilizing PFNA or IT devices [3].

PFNA has $6^{\circ}$ angles of the valgus, which is consistent with the proximal femoral anatomy. The proximal end of the main nail of IT adopts the trapezoidal cross section design and has a $4^{\circ}$ angle of the valgus. This type of design made it easy to insert the main nail during the operation [14]. The PFNA spiral blade provided the greatest degree surrounding the bone filling, which prevented the rotation of the fracture and implant cut-out [15]. IT with a unique combination of interlocking nails could generate a maximum of $15 \mathrm{~mm}$ of non-rotating axial compression. The design of the long tip and groove of the main nail of PFNA made its insertion more convenient and avoided the local concentration of stress
[16]. IT, with the distal bifurcation design of the main nail, dispersed the stress of the femoral anterior cortex, avoided periprosthetic fractures, and reduced the incidence of postoperative thigh pain $[14,21]$.

In addition, because of the intact femur anterior arch of elderly patients with osteoporotic IFs and the invariant intramedullary nail shape, if the main nail of the PFNA must be forced into the medullary cavity, it may cause the nail tip break through to the femoral anterior cortex or result in the risk of fracture $[22,23]$. We encountered difficulties with the insertion of the main nail, which was considered to have a significant relationship with the angle of the anterior arch of the femur. Postoperative thigh pain was observed between groups. The possible explanation was that the distal intramedullary 


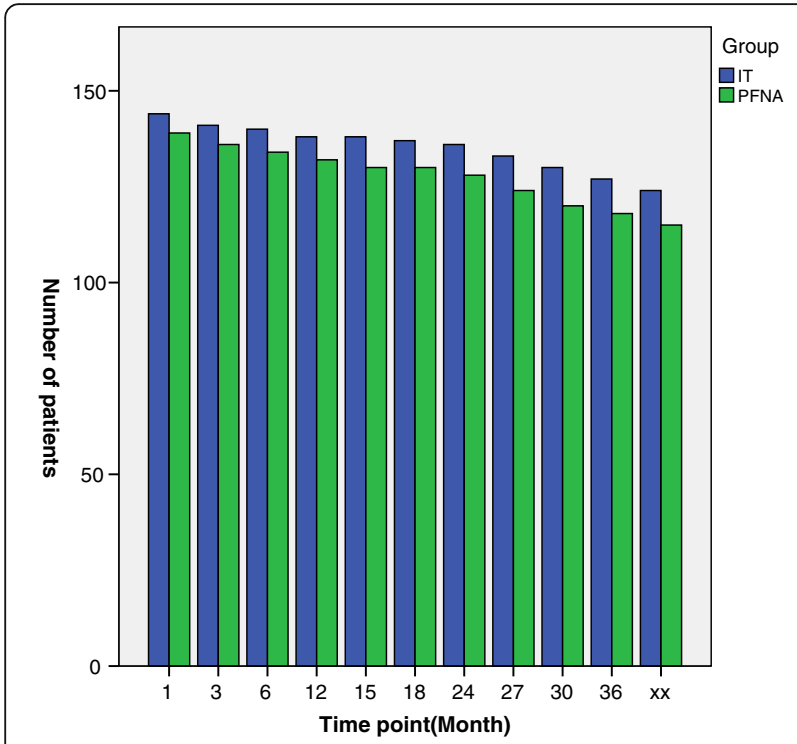

Fig. 2 The bar chart clearly shows the changes of the number of patients being lost to follow-up at each time point. The " $x x^{\prime}$ on the bar chart represents "final follow-up"

nail contacted the femur anterior cortex to generate friction, resulting in stress concentration, but no iatrogenic fracture was noted in the recent follow-ups [22, 24]. de Landevoisin et al. [25] reported 102 PFNA-treated cases and discovered one with acetabular penetration, 2 nailrelated fractures, and 5 blade back-outs $(2 \mathrm{~mm})$ responsible for pain, but there was no significant effect on post-reduction maintenance and fracture healing.

Although TAD is the strongest predictor of the cutout of a lag screw, increased age and osteoporosis might also be considered strong predictors. Some previously published studies underlined TAD as a predictor of implant failure [16, 21-23]. However, these studies failed to explore the relationship of age and osteoporosis to each implant category [16, 26].

Although intramedullary implants are better than conventional extramedullary implants, still they carry the variable rate of complications in form of femoral shaft fractures distal or around the tip of the main nail, cutout, more revision surgeries, and finally decreased functional outcome $[21,25]$. In the current study, 12 (8.6\%) observed for PFNA had femoral shaft fractures distal or

Table 1 The number of patients being lost to follow-up at each time point

\begin{tabular}{llllllllllll}
\hline & 1 & 3 & 6 & 12 & 15 & 18 & 24 & 27 & 30 & 36 & $F$ \\
\hline IT & 144 & 141 & 140 & 138 & 138 & 137 & 136 & 133 & 130 & 127 & 124 \\
PFNA & 139 & 136 & 134 & 132 & 130 & 130 & 128 & 124 & 120 & 118 & 115 \\
$N$ & 0 & 6 & 3 & 4 & 2 & 1 & 3 & 7 & 7 & 5 & 6 \\
\hline \multicolumn{7}{l}{$N$ the number of patients being lost to follow-up, $F$ final follow-up }
\end{tabular}

$N$ the number of patients being lost to follow-up, $F$ final follow-up
Table 2 Patient demographics and outcomes

\begin{tabular}{|c|c|c|c|}
\hline Variable & $\mathrm{IT}^{\mathrm{a}}(n=144)$ & $\operatorname{PFNA}^{\mathrm{b}}(n=139)$ & $P$ value \\
\hline Sex, no. M/F & $64 / 80$ & $53 / 86$ & $0.281^{* c}$ \\
\hline Age (years) & & & $0.839^{* d}$ \\
\hline $65-69$ & 40 & 36 & \\
\hline $70-79$ & 76 & 74 & \\
\hline $80-89$ & 22 & 20 & \\
\hline $90-92$ & 6 & 9 & \\
\hline $\mathrm{BMl}, \mathrm{kg} / \mathrm{m}^{2}$ & $26.1 \pm 2.1$ & $25.7 \pm 1.9$ & $0.130^{*}$ \\
\hline BMD & $-3.7 \pm 0.3$ & $-3.7 \pm 0.4$ & $0.179^{* e}$ \\
\hline Medical complications & 9 & 7 & $0.658^{* c}$ \\
\hline Side, no. Left/right & $81 / 63$ & $72 / 67$ & $0.453^{* c}$ \\
\hline OTA fracture type, no. & & & $0.679^{* d}$ \\
\hline $31 \mathrm{~A} 2.1$ & 44 & 36 & \\
\hline $31 \mathrm{~A} 2.2$ & 72 & 75 & \\
\hline $31 \mathrm{~A} 2.3$ & 28 & 28 & \\
\hline ASA classification & & & $0.501^{* d}$ \\
\hline 1 & 23 & 25 & \\
\hline 2 & 65 & 57 & \\
\hline 3 & 49 & 54 & \\
\hline 4 & 7 & 3 & \\
\hline Injury operation interval & & & $0.702^{* d}$ \\
\hline$<24 \mathrm{~h}$ & 22 & 23 & \\
\hline $24-48 \mathrm{~h}$ & 77 & 79 & \\
\hline $48-72 \mathrm{~h}$ & 29 & 27 & \\
\hline$>72 \mathrm{~h}$ & 16 & 10 & \\
\hline Follow-up (months) & $38.7 \pm 3.55$ & $39.1 \pm 3.43$ & $0.408^{* e}$ \\
\hline
\end{tabular}

IT InterTAN nail, PFNA proximal femoral nail anti-rotation, ASA American Society of Anesthesiologists, $B M I$ body mass index, $B M D$ bone mineral density *No statistically significant values

${ }^{\text {a} S}$ Smith \& Nephew, Memphis, Tennessee

bynthes, Solothurn, Switzerland

'Analyzed using chi-square test

${ }^{\mathrm{d}}$ Analyzed using the Mann-Whitney test

${ }^{\mathrm{e}}$ Analyzed using independent samples $t$-test

around the tip of the main nail. The high shaft fracture rate, despite its theoretically better design, was likely to be attributed to this fact that the looseness of distal locking screws existed, which was confirmed by the second revision surgery and would result in stress concentration at the tip of the main nail. The underlying cause may be osteoporosis, which results in the looseness of the PFNA fixation. In contrast, this high femoral shaft fracture rate is less observed in IT devices. Also, 11 cut-outs were observed in the PFNA-treated patients with osteoporosis. In patients with early postoperative excessive activity related to cut-outs, we removed the PFNA device and implemented traction treatment. Based on the outcome of comparative analysis, no statistically significant differences existed between groups in terms of fracture healing or HHS. However, the IT device was superior to the 
Table 3 Operation variables

\begin{tabular}{llll}
\hline Variable & $T^{\mathrm{a}}(n=144)$ & $\mathrm{PFNA}^{\mathrm{b}}(n=139)$ & $P$ value \\
\hline Reduction results, no. & & & $0.552^{* \mathrm{c}}$ \\
Anatomical & 82 & 84 & \\
Acceptable & 62 & 55 & \\
Poor & 0 & 0 & $0.948^{* \mathrm{c}}$ \\
Implant position, no. & & & \\
Optimal & 101 & 97 & \\
Suboptimal & 43 & 42 & $0.144^{* d}$ \\
TAD, mm & $19.9 \pm 1.25$ & $20.2 \pm 1.36$ & $0.21^{* d}$ \\
Length of stay (days) & $11.9 \pm 1.62$ & $11.67 \pm 1.88$ & $0.848^{* d}$ \\
Operation time (min) & $67.2 \pm 3.98$ & $68.9 \pm 4.27$ & $0.078^{* d}$ \\
Blood loss (ml) & $180.7 \pm 23.03$ & $185.1 \pm 18.96$ &
\end{tabular}

TAD tip-apex distance, IT InterTAN nail, PFNA proximal femoral nail anti-rotation

*No statistically significant values

${ }^{\text {a }}$ Smith \& Nephew, Memphis, Tennessee

${ }^{b}$ Synthes, Solothurn, Switzerland

'Analyzed using the Mann-Whitney test

${ }^{d}$ Analyzed using independent samples $t$-test

PFNA device in these aspects (cut-out, femoral shaft fracture distal or around the tip of the main nail, varus collapse of the femoral head, and postoperative thigh pain), which could be considered an important factor when deciding the superiority of any particular implant.

Caution needs to be taken when interpreting relatively high failure rates of internal fixation in the current study, as several factors have been identified. Firstly, stable fracture patterns in osteoporotic bone in conjunction with the quality of the reduction and screw positioning in the femoral head-neck fragment did not predispose to implant cut-out, femoral shaft fracture, or varus collapse of the femoral head. Often the failure is due to inadequate reduction, poorly placed femoral head-neck screw, or mal-positioning of the implant. Of the 38 cases of failure in our series, three fractures were poorly reduced, six femoral head-neck screws were poorly placed, and three main nails were inclined to the lateral wall. Secondly, early functional exercise and high activity levels might increase the risk of implant failure. Appropriate delayed partial weight-bearing might have a strong positive effect on the outcome. Thirdly, patient ages and time to surgery played an important role in fragility fracture treatment. We noted implant failure tended to occur in patients aged 80 years or older or patients with surgical intervention time more than $72 \mathrm{~h}$ in our study. Fourthly, TAD less than $19 \mathrm{~mm}$ might be associated with failure rates. The finding might provide a guide for surgeons in optimal screw position for reducing the risk of mechanical failure when performing reduction and fixation of IFs.

Our study is limited by its retrospective nature. Furthermore, patient- and surgeon-related confounders may have existed. Nevertheless, both groups were well matched, which allowed us to draw the conclusion that the between-implant differences observed were not related to the patients' demographics or severity of fracture.

Table 4 Long-term follow-up outcomes

\begin{tabular}{|c|c|c|c|}
\hline Variable & $\mathrm{IT}^{\mathrm{a}}(n=144)$ & $\operatorname{PFNA}^{\mathrm{b}}(n=139)$ & $P$ value \\
\hline Screw cut-out & 3 & 11 & $0.024^{* c}$ \\
\hline Femoral shaft fracture ${ }^{\#}$ & 3 & 12 & $0.014^{* c}$ \\
\hline Delayed union & 4 & 5 & $0.709^{c}$ \\
\hline Malunion & 2 & 0 & $0.498^{d}$ \\
\hline Non-union & 2 & 0 & $0.498^{d}$ \\
\hline Varus collapse of the femoral head & 1 & 8 & $0.020^{* c}$ \\
\hline Lower limb shortening $(>1.5 \mathrm{~cm})$ & 2 & 2 & $0.972^{c}$ \\
\hline Heterotopic ossification & 3 & 1 & $0.326^{c}$ \\
\hline Avascular necrosis of the femoral head & 1 & 1 & $1.000^{d}$ \\
\hline Postoperative thigh pain & 5 & 13 & $0.043^{* c}$ \\
\hline Lower extremity venous thrombosis & 7 & 6 & $0.797^{c}$ \\
\hline $\mathrm{HHS}$ at the last follow-up & $72.2 \pm 7.27$ & $72.4 \pm 7.20$ & $0.842^{e}$ \\
\hline Mortality & 19 & 22 & $0.529^{c}$ \\
\hline
\end{tabular}

IT InterTAN nail, PFNA proximal femoral nail anti-rotation, HHS Harris Hip Score

*Statistically significant values

\#Displaced shaft fractures distal or around the tip of the main nail

${ }^{\text {a }}$ Smith \& Nephew, Memphis, Tennessee

${ }^{\text {b}}$ Synthes, Solothurn, Switzerland

CAnalyzed using the Pearson chi-squared test

${ }^{d}$ Analyzed using Fisher's exact test

${ }^{\mathrm{e}}$ Analyzed using independent samples $t$-test 


\section{Conclusions}

There is a compelling evidence that IT might be more appropriate in stabilizing osteoporotic IFs (OTA 3.1A2.1A2.3). Although we have shown a higher complication rate than in some previous series, the application of IT for the fixation of osteoporotic IFs (OTA 3.1A2.1-A2.3) might be the preferred choice compared with PFNA. However, the undeniable fact is that the current study was unable to reveal whether the IT or the PFNA is the optimal treatment for osteoporotic IFs (OTA 3.1A2.1-A2.3). As such, the authors recommend that further prospective randomized trials would be mandatory.

\section{Abbreviations \\ ASA: American Society of Anesthesiologists; BMD: Bone mineral density; CPM: Continuous passive motion; HHS: Harris Hip Score; IFs: Intertrochanteric fractures; IT: InterTAN nail; PFNA: Proximal femoral nail anti-rotation; SD: Standard deviation; TAD: Tip-apex distance}

\section{Acknowledgements}

The authors would like to acknowledge Dr. Lili Zhang for his assistance with the technical help.

\section{Funding}

No funding was received for this study or for the writing of this manuscript.

\section{Availability of data and materials}

The datasets supporting the conclusions of this article are included within the article.

\section{Authors' contributions}

$\mathrm{HZ}$ performed the data collection and analysis and participated in manuscript writing. $X X Z, P X, X S Z$, and NZ performed the database setup and statistical analysis. GP, WY, XCZ, XSZ, and DC performed the operations. XCZ participated in the study design and coordination and helped to draft the manuscript. All of the authors have read and approved the final manuscript.

\section{Ethics approval and consent to participate}

The study was approved by the institutional ethics review board of the First Affiliated Hospital, Sun Yat-sen University, and an exemption for informed consent was obtained from our Investigational Ethical Review Board.

\section{Consent for publication}

An exemption from informed consent was obtained from our responsible Investigational Ethical Review Board.

\section{Competing interests}

The authors declare that they have no competing interests.

\section{Publisher's Note}

Springer Nature remains neutral with regard to jurisdictional claims in published maps and institutional affiliations.

\section{Author details}

${ }^{1}$ Emergency Department, The First Affiliated Hospital of Sun Yat-sen University, Huangpu East Road No. 183, Huangpu District, Guangzhou, Guangdong 510700, China. ${ }^{2}$ Endocrine Department, The First Affiliated Hospital of Sun Yat-sen University, Huangpu East Road No. 183, Huangpu District, Guangzhou, Guangdong 510700, China. ${ }^{3}$ Department of ENT, The First Affiliated Hospital of Sun Yat-sen University, Huangpu East Road No. 183, Huangpu District, Guangzhou, Guangdong 510700, China. ${ }^{4}$ Department of Orthopedics, The First Affiliated Hospital of Sun Yat-sen University, Huangpu East Road No. 183, Huangpu District, Guangzhou, Guangdong 510700, China. ${ }^{5}$ Department of Anesthesiology, The First Affiliated Hospital of Sun Yat-sen University, Huangpu East Road No. 183, Huangpu District, Guangzhou, Guangdong 510700, China. ${ }^{6}$ Radiology Department, The First Affiliated Hospital of Sun Yat-sen University, Huangpu East Road No. 183,
Huangpu District, Guangzhou, Guangdong 510700, China. ${ }^{\text {DDepartment of }}$ Joint Surgery, The First People's Hospital of Jingmen, Xiangshan Avenue No. 168, Dongbao District, Jingmen, Hubei 448000, China. ${ }^{8}$ Department of Orthopaedics, Jinshan Hospital, Fudan University, Longhang Road No. 1508 , Jinshan District, Shanghai City 201508, China.

Received: 25 March 2017 Accepted: 25 September 2017

Published online: 10 October 2017

\section{References}

1. PRJ P, Lord R, Jawad A, Dawe E, Stott P, Rogers B, Gill R. Changing trends in the management of intertrochanteric hip fractures - a single centre experience. Injury. 2016;47(7):1525-9.

2. Kanakaris NK, Tosounidis TH, Giannoudis PV. Nailing intertrochanteric hip fractures: short versus long; locked versus nonlocked. J Orthop Trauma. 2015;29:S10-6

3. Seyhan M, Turkmen I, Unay K, Ozkut AT. Do PFNA devices and Intertan nails both have the same effects in the treatment of trochanteric fractures? A prospective clinical study. J Orthop Sci. 2015;20(6):1053-61.

4. Li MH, Wu L, Liu Y, Wang CM. Clinical evaluation of the Asian proximal femur intramedullary nail antirotation system (PFNA-II) for treatment of intertrochanteric fractures. J Orthop Surg Res. 2014;9

5. Zha G-C, Chen Z-L, Qi X-B, Sun J-Y. Treatment of pertrochanteric fractures with a proximal femur locking compression plate. Injury. 2011;42(11):1294-9.

6. Henzman C, Ong K, Lau E, Seligson D, Roberts CS, Malkani AL. Complication risk after treatment of intertrochanteric hip fractures in the Medicare population. Orthopedics. 2015;38(9):E799-805.

7. Kleweno C, Morgan J, Redshaw J, Harris M, Rodriguez E, Zurakowski D, Vrahas M, Appleton P. Short versus long cephalomedullary nails for the treatment of intertrochanteric hip fractures in patients older than 65 years. J Orthop Trauma. 2014;28(7):391-7.

8. Boone C, Carlberg KN, Koueiter DM, Baker KC, Sadowski J, Wiater PJ, Nowinski GP, Grant KD. Short versus long intramedullary nails for treatment of intertrochanteric femur fractures (OTA 31-A1 and A2). J Orthop Trauma. 2014:28(5):E96-E100

9. Wang $X, Y u$ G-R. Intramedullary nails result in more reoperations than sliding hip screws in two-part intertrochanteric fractures. Clin Orthop Relat Res. 2013;471(7):2408-10

10. Menezes DFA, Gamulin A, Noesberger B. Is the proximal femoral nail a suitable implant for treatment of all trochanteric fractures? Clin orthop relat research. 2005:439:221-7.

11. Kim WY, Han CH, Park JI, Kim JY. Failure of intertrochanteric fracture fixation with a dynamic hip screw in relation to pre-operative fracture stability and osteoporosis. Int Orthop. 2001;25(6):360-2.

12. Shayesteh-Azar M, Karimi-Nasab MH, Sajjadi-Saravi M, Daneshpoor SMM Hadian A, Olia G. Comparison of surgical complications of intertrochanteric hip fractures between non-osteoporotic and osteoporotic patients. J Pak Med Assoc. 2013:63(6):752-5.

13. Huang TW, Chuang PY, Lin SJ, Lee CY, Huang KC, Shih HN, Lee MS, Hsu RWW, Shen WJ. Teriparatide improves fracture healing and early functional recovery in treatment of osteoporotic intertrochanteric fractures. Medicine. 2016:95(19):e3626

14. Yu W, Zhang $X$, Zhu $X, \mathrm{Hu}$ J, Liu Y. A retrospective analysis of the InterTan nail and proximal femoral nail anti-rotation-Asia in the treatment of unstable intertrochanteric femur fractures in the elderly. J Orthop Surg Res. 2016;11

15. Hartford JM, Patel A, Powell J. Intertrochanteric osteotomy using a dynamic hip screw for femoral neck nonunion. J Orthop Trauma. 2005;19(5):329-33.

16. Zhang S, Zhang KR, Jia YF, Yu B, Feng W. InterTan nail versus proximal femoral nail antirotation-Asia in the treatment of unstable trochanteric fractures. Orthopedics. 2013;36(3):E288-94.

17. Mereddy P, Kamath S, Ramakrishnan M, Malik H, Donnachie N. The AO/ASIF proximal femoral nail antirotation (PFNA): a new design for the treatment of unstable proximal femoral fractures. Injury. 2009;40(4):428-32.

18. Ruecker AH, Rupprecht M, Gruber M, Gebauer M, Barvencik F, Briem D, Rueger JM. The treatment of intertrochanteric fractures: results using an intramedullary nail with integrated cephalocervical screws and linear compression. J Orthop Trauma. 2009;23(1):22-30.

19. Li S, Chang SM, Jin YM, Zhang YQ, Niu WX, Du SC, et al. A mathematical simulation of the tip-apex distance and the calcar-referenced tip-apex 
distance for intertrochanteric fractures reduced with lag screws. Injury. 2016; 47(6):1302-8.

20. Santoni BG, Nayak AN, Cooper SA, Smithson IR, Cox JL, Marberry ST, Sanders RW. Comparison of femoral head rotation and varus collapse between a single lag screw and integrated dual screw intertrochanteric hip fracture fixation device using a cadaveric Hemi-Pelvis biomechanical model. J Orthop Trauma. 2016;30(4):164-9.

21. Rupprecht M, Grossterlinden L, Sellenschloh K, Hoffmann M, Puschel K, Morlock M, Rueger JM, Lehmann W. Internal fixation of femoral neck fractures with posterior comminution. A biomechanical comparison of DHS (R) and Intertan nail (R). Int Orthop. 2011;35(11):1695-701.

22. Nikoloski AN, Osbrough AL, Yates PJ. Should the tip-apex distance (TAD) rule be modified for the proximal femoral nail antirotation (PFNA)? A retrospective study. J Orthop Surg Res. 2013;8

23. Raviraj A, Anand A, Chakravarthy M, Pai S. Proximal femoral nail antirotation (PFNA) for treatment of osteoporotic proximal femoral fractures. European $J$ Orthop Surg Traumatol. 2012;22(4):301-5.

24. Niikura T, Lee SY, Matsumoto T, Fukui T, Kawakami Y, Akisue T, Kuroda R, Kurosaka M. Backout of the helical blade of proximal femoral nail antirotation and accompanying fracture nonunion. Orthopedics. 2012;35(8):E1264-6.

25. de Landevoisin ES, Bertani A, Candoni P, Charpail C, Demortiere E. Proximal femoral nail antirotation (PFN-ATM) fixation of extra-capsular proximal femoral fractures in the elderly: retrospective study in 102 patients. Orthop Traumatol Surg Res. 2012;98(3):288-95.

26. Andruszkow H, Frink M, Fromke C, Matityahu A, Zeckey C, Mommsen P, Suntardjo S, Krettek C, Hildebrand F. Tip apex distance, hip screw placement, and neck shaft angle as potential risk factors for cut-out failure of hip screws after surgical treatment of intertrochanteric fractures. Int Orthop. 2012;36(11):2347-54

\section{Submit your next manuscript to BioMed Central and we will help you at every step:}

- We accept pre-submission inquiries

- Our selector tool helps you to find the most relevant journal

- We provide round the clock customer support

- Convenient online submission

- Thorough peer review

- Inclusion in PubMed and all major indexing services

- Maximum visibility for your research

Submit your manuscript at www.biomedcentral.com/submit

) Biomed Central 